\title{
Aproximaciones Temáticas entre la Academia de Arquitectura en la UAEH, el Diseño Edilicio Básico y el Colegio de Arquitectos de Hidalgo
}

\section{Thematic Approaches between the Academy of Architecture at UAEH, Basic Edilicio Design and the College of Architects of Hidalgo}

\author{
Continente Elizalde-Domínguez \\ Área Académica de Ingeniería y Arquitectura del Instituto de Ciencias Básicas e Ingeniería de la Universidad Autónoma del Estado de Hidalgo.
}

\begin{abstract}
Resumen.
A continuación se presenta el mapa de relaciones temáticas establecido entre la Academia de Arquitectura en la Universidad Autónoma del Estado de Hidalgo, el diseño edilicio básico y el Colegio de Arquitectos de Hidalgo A.C. Obtenido mediante la investigación con enfoque cualitativo de tipo transeccional no experimental de alcance exploratorio y axial. Desarrollada para analizar aleatoriamente diversas fuentes informativas y documentales, bajo la premisa de señalar los elementos constitutivos de estos tres nodos, codificarlos y jerarquizarlos en términos de beneficio para las condiciones mercantiles del ejercicio proyectual en la Zona de Pachuca. La estructura gráfica del mapa alude a la hipótesis resultante, es holística e implica la factibilidad de su aplicación en la toma de conciencia, decisiones y gestión de directivas; concernientes a la generalidad local de las entidades educativas, de investigación y del gremio pero específicamente respecto del presente y futuro de las oportunidades de ingreso financiero propias del empleo y autoempleo.
\end{abstract}

Palabras Clave:

Arquitectura en la UAEH, diseño edilicio básico, Colegio de Arquitectos de Hidalgo A.C.

\begin{abstract}
.
The following is the map of thematic relationships established between the Academy of Architecture at the Autonomous University of the State of Hidalgo, the basic edilicio design and the College of Architects of Hidalgo A.C. Obtained through research with a qualitative non-experimental transectional approach of exploratory and axial scope. Developed to randomly analyze various informative and documentary sources, under the premise of pointing out the constituent elements of these three nodes, coding them and ranking them in terms of benefit for the commercial conditions of the project exercise in the Pachuca Zone. The graphic structure of the map alludes to the resulting hypothesis, it is holistic and implies the feasibility of its application in the taking of awareness, decisions and management of directives concerning the local generality of the educational, research and union entities but specifically regarding the present and future of financial income opportunities typical of employment and self-employment.
\end{abstract}

Keywords:

Architecture in the UAEH, basic edilicio design, Colegio de Arquitectos de Hidalgo A.C.

\section{Introducción}

El presente estudio fue realizado para dar soporte a la ponencia homónima, correspondiente al "XX Seminario de Investigación del Área Académica de Ingeniería y Arquitectura", programado en modalidad virtual para los días 11 y 12 de noviembre del 2020 con trasmisión en vivo mediante la fangage del Instituto de Ciencias Básicas e Ingeniería de la Universidad Autónoma del Estado de Hidalgo. Destacado con el lema "20 años de impacto en el sector productivo del país atendiendo las necesidades académicas, tecnológicas y de confort de la sociedad", divulgado en los medios informáticos con el anuncio alusivo, ver Figura 1.

Área académica que alberga en el nivel superior a los programas educativos de las licenciaturas en ingeniería industrial, civil y arquitectura. Además de las maestrías en ciencias de la ingeniería industrial y de manufactura. Así como el diplomado y el doctorado en ingeniería industrial (AAIyA, 2020).

$\mathrm{La}$ ponencia se articula al interior del proyecto de investigación, denominado como Evaluación de Neo Repentinas, clave UAEH-DI-17-ICBI-INGSF29, con la finalidad de incrementar la divulgación de sus productos, publicados en libros

*Autor para la correspondencia: profe_6389@uaeh.edu.mx

Correo electrónico: profe_6389@uaeh.edu.mx (Elizalde-Domínguez, Continente). 
y artículos, pero alternativamente para fijar puntos de vista relacionados localmente al medio profesional y académico del diseño edilicio en general y de manera específica al diseño edilicio básico.

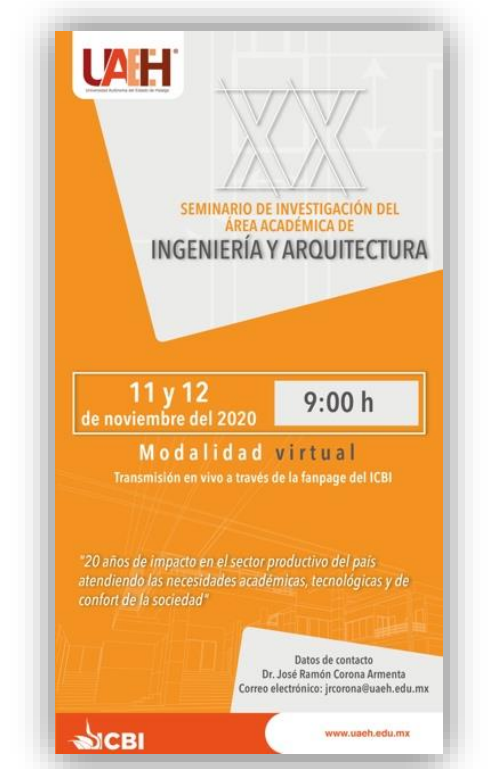

Figura 1: Anuncio del seminario; Fuente: AAIyA.

Puntos de vista considerados como oportunidades idóneas para influir en el abanico de reflexiones, toma de decisiones o gestión de directivas relativas a las entidades educativas, de investigación y gremial.

\section{2. Ámbito local.}

Se integra por la zona geográfica de estudio representada por el tejido urbano de la ciudad de Pachuca, las escuelas de arquitectura localizadas en la zona o cercanas a ella, el Colegio de Arquitectos de Hidalgo A.C. con su arancel y las acciones de divulgación del diseño edilicio básico. Asimismo por la definición de los beneficios para las condiciones mercantiles del ejercicio proyectual.

\subsection{La Zona de Estudio.}

Se trata de la extensión territorial comprendida contundentemente entre los municipios de Pachuca de Soto, Mineral de la Reforma, Mineral del Chico, Mineral del Monte y Zempoala. Sutilmente entre los municipios de Zapotlán de Juárez y San Agustín Tlaxiaca. Dividida en cuatro cuadrantes con una superficie de $124.307 \mathrm{~km}^{2}$ y un perímetro de $128.79 \mathrm{~m}$ (Elizalde, 2019a). Alojada en el estado de Hidalgo, caracterizado por ocupar el $1.06 \%$ de la superficie del territorio nacional y contener al $2.4 \%$ de su población con 2`858,359 habitantes, de la cual el 52\% es urbana y el $48 \%$ es rural. Donde el sector del comercio es el que más aporta al PIB con 1.7\% (INEGI, 2020). Mientras la industria de la construcción, actividad laboral próxima al desempeño profesional del diseño edilicio, presenta un valor total de producción igual a $\$ 13^{\prime} 125,833$. Generados en la entidad por las empresas dedicadas a la edificación. Parámetro protagonizado por la “edificación”, tipo de obra con valor productivo de \$3'336,003; superior a otros como "agua, riego y saneamiento" con $\$ 351,211$; asimismo "electricidad y telecomunicaciones" con \$2'796,487 entre otros (INEGI, 2016).
De la superficie estatal igual a $20,813 \mathrm{~km}^{2}$ (INEGI, 1991) Pachuca de Soto ocupa el $0.74 \%$ (INEGI, 2009a), Mineral de la Reforma el 0.56\% (INEGI, 2009b), Mineral del Monte el 0.26\% (INEGI, 2009c), Mineral del Chico el 0.92\% (INEGI, 2009d), Zempoala el 1.54\% (INEGI, 2009e), Zapotlán de Juárez el 0.56\% (INEGI, 2009f) y San Agustín Tlaxiaca el 1.45\% (INEGI, 2009g). En otros datos, el municipio de Pachuca de Soto, posee un 53.35 en el Índice Básico de las Ciudades Prosperas o CPI, que le coloca en la escala global de prosperidad como "moderadamente débil". Dentro de la "aglomeración de Pachuca", ver Figura 2, este municipio presenta en la última década un patrón expansivo de urbanización. Correspondiente a una tasa de crecimiento anual de viviendas de $8.4 \%$, superior a la tasa anual de crecimiento de la población de 3.5\% (ONU-HABITAT, 2016).

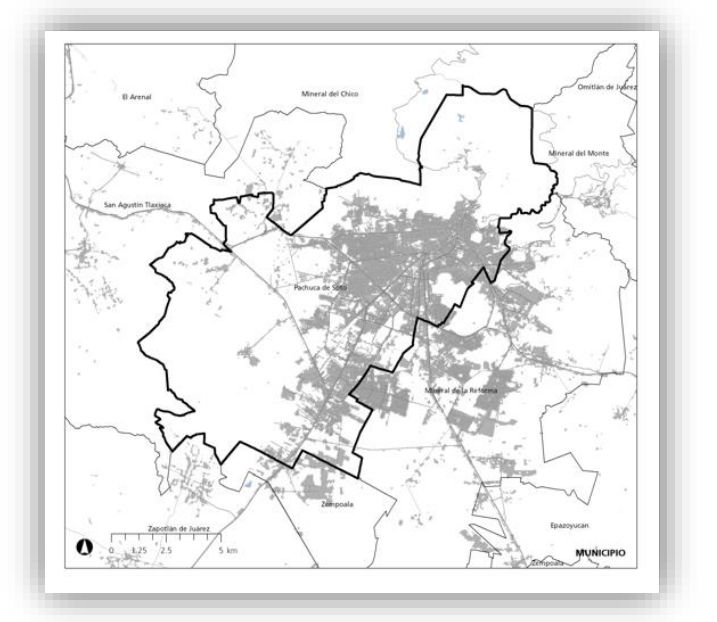

Figura 2: Aglomeración de Pachuca; Fuente: CONAPO.

Como integrante de la "zona metropolitana de Pachuca" el mismo municipio se encuentra catalogado con la clave 13048, posee una superficie de $154.1 \mathrm{~km}^{2}$, densidad urbana de $86.4 \mathrm{hab} / \mathrm{ha}$, población de 277,375 habitantes y una tasa de crecimiento de $0.7 \%$ en $2010-2015$, a la baja según las tasas de crecimiento presentadas en $1990-2000$ y $2000-2010$, de $3.1 \%$ y $0.9 \%$ respectivamente (SEDATU-CONAPO-INEGI, 2018). En esta zona la legalidad de las facultades como la proyectual de la arquitectura y su materialización en obra son normalizadas por la Ley del Ejercicio Profesional para el Estado de Hidalgo (LEPEH, 2001) y por la Ley de Asentamientos Humanos, Desarrollo Urbano y Ordenamiento Territorial del Estado de Hidalgo (LAHDUOTEH, 2018), general y respectivamente.

\subsection{Las escuelas de arquitectura.}

Con el propósito de convocar a las escuelas de arquitectura, cercanas o en la zona de estudio, para que solicitaran la conferencia "Las Competencias Profesionalizantes Estudiantiles y Docentes en el Diseño Edilicio Básico". Fueron entregados, por parte del proyecto de investigación para la Evaluación de Neo Repentinas, 13 oficios de invitación a finales del 2018, presentada en una sola ocasión, ver Figura 3. Situación adecuada para registrar 8 entidades educativas de nivel licenciatura $(l)$ en el municipio (Mpio) de Pachuca de Soto $(P), 2$ en el Mpio. de Mineral de la Reforma $(M), 2$ en el Mpio. de San Agustín Tlaxiaca $(S)$ y 1 en el Mpio. de Tolcayuca $(T)$ con enfoque bioclimático, Tabla 1.

La conferencia tenía el cometido directo de informar sobre el "curso con concurso" gratuito y el premio de $\$ 5,000.00$ o 
$\$ 10,000.00$. Para participar se requería de un oficio institucional de solicitud por estudiante donde se debía incluir algún docente.

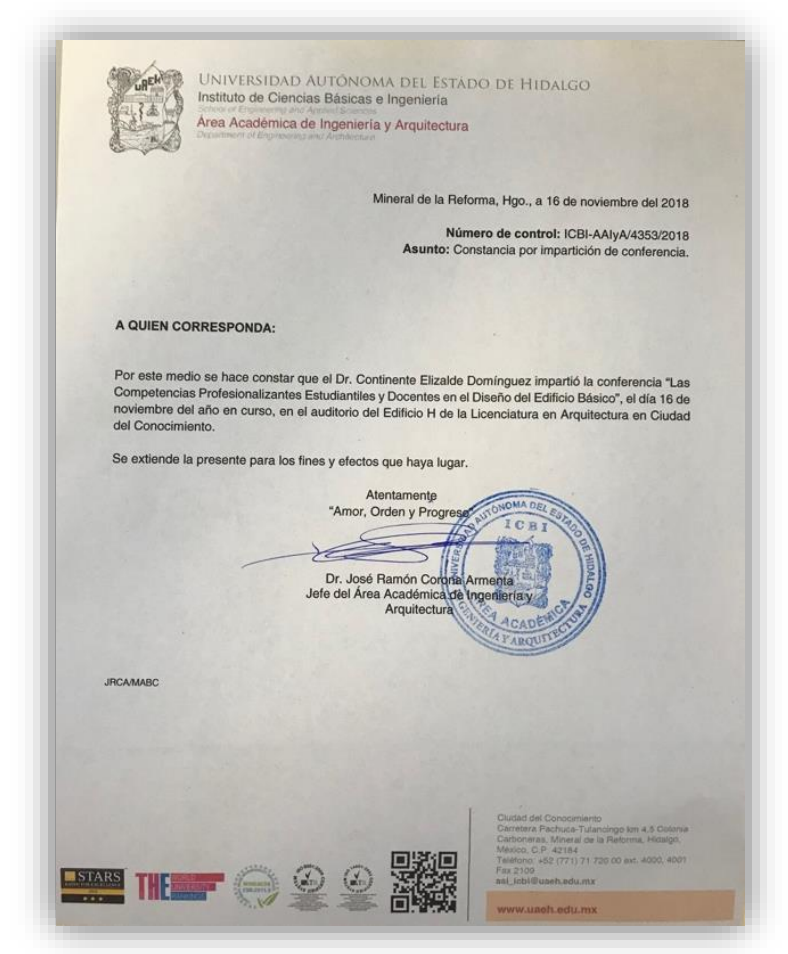

Figura 3: Constancia de la conferencia; Fuente: AAIyA.

El contenido comprendía las generalidades de las "repentinas y competencias", la explicación de las "competencias estudiantiles" y las "competencias docentes", la definición del "diseño edilicio básico", la participación del proyecto de investigación para la Evaluación de Neo Repentinas, la aplicación del Modelado Arquitectónico Concurrente (Elizalde, 2019b) y del Detonante Gráfico (Elizalde, 2019c), la integración física de la "carpeta de trabajo" y de las dinámicas del "curso con concurso" desarrollado a modo de taller.

Tabla 1: Escuelas de arquitectura cercanas o en la zona de estudio.

\begin{tabular}{|c|c|c|c|}
\hline $\mathrm{N}$ & Escuelas de Arquitectura & Mpio & Nivel \\
\hline 1 & Instituto Tecnológico de Pachuca & $P$ & $l$ \\
\hline 2 & $\begin{array}{l}\text { Instituto Tecnológico y de Estudios } \\
\text { Superiores de Monterrey }\end{array}$ & $P$ & $l$ \\
\hline 3 & Universidad de Durango & $P$ & $l$ \\
\hline 4 & $\begin{array}{c}\text { Universidad Interamericana para el } \\
\text { Desarrollo }\end{array}$ & $P$ & $l$ \\
\hline 5 & Centro de Estudios Superiores Antares & $P$ & $l$ \\
\hline 6 & Centro Universitario Continental & $P$ & $l$ \\
\hline 7 & $\begin{array}{c}\text { Centro Universitario Metropolitano } \\
\text { Hidalgo }\end{array}$ & $P$ & $l$ \\
\hline 8 & $\begin{array}{c}\text { Centro de Estudios Universitarios de } \\
\text { Hidalgo }\end{array}$ & $P$ & $l$ \\
\hline 9 & $\begin{array}{l}\text { Universidad Autónoma del Estado de } \\
\text { Hidalgo }\end{array}$ & $M$ & $l$ \\
\hline 10 & Instituto Tecnológico Latinoamericano & $M$ & $l$ \\
\hline 11 & Universidad La Salle & $S$ & $l$ \\
\hline 12 & $\begin{array}{l}\text { Centro Cultural Europeo de Estudios } \\
\text { Universitarios de Hidalgo }\end{array}$ & $S$ & $l$ \\
\hline 13 & $\begin{array}{c}\text { Universidad Politécnica Metropolitana } \\
\text { de Hidalgo }\end{array}$ & $T$ & $l$ \\
\hline
\end{tabular}

Fuente: elaboración propia.

La capacidad esperada permitía la participación de 15 estudiantes de las diferentes escuelas invitadas y el premio se entregaría a los dos mejores anteproyectos ejecutivos, elegidos por el estudiantado participante. Se gestionó como sede al aula 6 del Área Académica de Ingeniería y Arquitectura, la duración se estimó para 42 sesiones en 21 semanas sumando 168 horas, la fecha de inicio se determinó para el martes 29 de enero del 2019 y la de término para el viernes 21 de junio del mismo 2019.

El horario, únicamente presencial, se programó para los martes y viernes de 7:30hrs a 11:30hrs. Las reglas para contender por el premio establecieron que únicamente se permitían 3 faltas, se debían desarrollar y entregar 2 "carpetas de trabajo", una por cada anteproyecto ejecutivo, en las fechas indicadas. Para el personal docente de las escuelas participantes, el curso-taller se previó como semipresencial y se fundamentó en el libro Programación Gantt para un Curso Estratégico de Diseño Edilicio Básico (Elizalde, 2019d).

Finalmente, los resultados esperados comprendían la recopilación de la información proveniente de los "rendimientos escolares y extraescolares", del apropiamiento de las "competencias escolares" y de las "competencias docentes". Desafortunadamente no se tuvieron inscripciones, circunstancia atribuida al carácter presencial y semipresencial del "curso con concurso". Con ello se alejó la oportunidad de visualizar el factible acercamiento con las habilidades blandas o "soft skills", necesarias para complementar perfiles empresariales deseables (Tito \& Serrano, 2016) concordantes con la "formación integral" de "la sociedad del conocimiento, las tecnologías y la comunicación" (Marrero et al, 2018).

\subsection{El Colegio de Arquitectos de Hidalgo y el arancel de la FCARM.}

La trascendencia gremial del Colegio de Arquitectos de Hidalgo A.C. (CAH) se remonta a 1965, año de su constitución mediante acta notarial, cuatro años después se fortalece con el decreto de la "Ley del Ejercicio Profesional para el Estado de Hidalgo" y posteriormente obtiene la cédula profesional del estado. Este colegio se supone como el primero del país porque se registra "con el No. 1 en el primer libro de actas de la FCARM". Entre sus órganos de gobierno se encuentra al comité en turno, atendiendo el periodo 2019-2021; a la junta de honor y al comité delegacional, este, con representatividad en los municipios de Apan, Mixquiahuala de Juárez, Tepeji del Río de Ocampo, Tula de Allende, Tulancingo y Zacualtipán de Ángeles. De sus órganos operativos, solo se tiene al patronato, y sobre la afiliación, se hace referencia a ciertos requisitos; como a la solicitud de ingreso y a la integración de un expediente. Físicamente se ubica en Av. Benito Juárez 1105, Col. Periodistas, C.P. 42060, Pachuca de Soto, Hgo. (CAH, 2020). Su logotipo le distingue en diferentes medios y eventos, ver Figura 4.

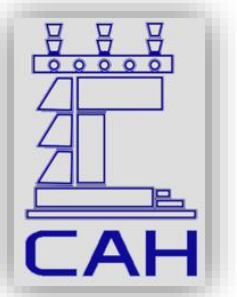

Figura 4: Logotipo del Colegio de Arquitectos de Hidalgo A.C.; Fuente: CAH. 
Su estatuto comprende misión, visión y objetivos, así como 91 artículos divididos en 5 capítulos. Son destacables aquellos aspectos de índole exógena como la intención de "contribuir al avance del hábitat humano dentro del Desarrollo Urbano para el servicio de la sociedad", contenido en la misión del colegio. De índole endógena al observarse como generador en la actividad profesional particular de "logros altamente competitivos y avances en los campos científicos" indicado en su visión. De territorialidad con la pretensión expresada en el objetivo general de agremiar a "arquitectos legalmente registrados y establecidos en el Estado de Hidalgo". Incluido el aspecto de incidencia mercantil del objetivo específico colocado en el quinto lugar, dispuesto a "identificar los ámbitos de oportunidades para los Arquitectos que les permitan un mejor ingreso para obtener un desarrollo profesional de acuerdo a sus intereses".

Su código de ética se integra por 11 artículos contenidos en un solo título y hace referencia a los deberes y obligaciones del gremio al respecto de la patria, la colectividad, el estado, la clientela y su familia, entre otros. El arancel refleja la mecánica para procurar el pago de los honorarios debidos al servicio de diseño e integración de los proyectos ejecutivos, incluidos los causados por visitas o consultas técnicas (ECA-CAH, 2012).

A nivel nacional, la Federación de Colegios de Arquitectos de la República Mexicana A.C. protocolizó el arancel ante notario para 2008. En él se hace mención a los servicios profesionales como el "diseño arquitectónico", "diseño urbano" y "consultorías".

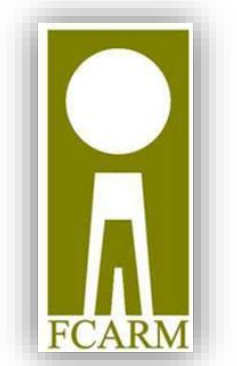

Figura 5: Logotipo de la Federación de Colegios de Arquitectos de la República Mexicana; Fuente: FCARM.

El Capítulo II precisa "los alcances particulares mínimos y ponderación porcentual", enlistados para fijar el "pago por cada etapa del desarrollo de un proyecto integral o por la contratación parcial". Divididos en 7 etapas clasificadas como "diseño conceptual" con el $11 \%$, "anteproyecto" $20 \%$, "diseño ejecutivo" $35 \%$, "estructura" $12 \%$, "instalación eléctrica" $10 \%$, "instalación hidrosanitaria" $8 \%$ e "instalación de gas" con $4 \%$ para un total del $100 \%$ (FCARM, 2020). Del mismo modo, su logotipo es su principal distintivo, ver Figura 5.

\subsection{La divulgación del diseño edilicio básico.}

El diseño edilicio básico fue definido en 2019 como un sector del quehacer proyectual tanto académico como profesional de la arquitectura que se concentra inicialmente al estudio y tratamiento resolutivo de 21 tipologías de edificaciones comerciales y de servicio franquisiatarias. Constituidas por 10 o 15 espacios interiores, dispuestos en 1 o 2 niveles de altura, desarrollables en extensiones de 150 a $600 \mathrm{~m}^{2}$ pero parametrizadas en factores de costo de 0.58 a 2.07 del arancel de la FCARM (Elizalde, 2020).

Pero su divulgación directa e indirecta comienza desde el año 2013 y se ha manifestado hasta el 2020 de manera personal síncrona o asíncrona en diversos medios: 13 conferencias $\left({ }^{c l}\right), 6$ presentaciones de libros o exhibiciones de trabajo $\left.{ }^{(p I}\right), 11$ ponencias $\left({ }^{\left({ }^{2}\right.}\right)$ y 2 seminarios doctorales $\left({ }^{s d}\right)$. Para un compendio superior a 30 eventos, Tabla 2.

Tabla 2: Divulgación del diseño edilicio básico.

\begin{tabular}{|c|c|c|}
\hline $\mathrm{N}$ & Titulo & Año \\
\hline 1,2 & $\begin{array}{c}\text { Arquitectura e Identidad a } \\
\text { través del Tiempo } \\
\boldsymbol{c l}\end{array}$ & $2013 / 14$ \\
\hline 3 & $\begin{array}{c}\text { El Modelado Eventual de los Continentes } \\
\text { Arquitectónicos } c \boldsymbol{c}\end{array}$ & 2014 \\
\hline 4 & $\begin{array}{l}\text { Presentación de las Carpetas de Trabajo } \\
\text { como } \\
\text { resultados del diseño edilicio básico }\end{array}$ & 2015 \\
\hline 5 & $\begin{array}{l}\text { Sistema Cuali-Cuantitativo Establecido } \\
\text { para Valorar las Propuestas Provenientes } \\
\text { del Diseño Arquitectónico Edilicio }{ }^{c l}\end{array}$ & 2015 \\
\hline 6,7 & $\begin{array}{c}\text { Nicho de Mercado en el Diseño } \\
\text { Arquitectónico } \\
c \boldsymbol{c}\end{array}$ & 2015 \\
\hline 8 & Visión para el Estudio de la Arquitectura & 2015 \\
\hline 9 & $\begin{array}{l}\text { Sistema para Valorar la Complejidad del } \\
\text { Diseño Arquitectónico Edilicio } \\
c l\end{array}$ & 2016 \\
\hline 10 & $\begin{array}{l}\text { La Libertad Edilicia como Detonante del } \\
\text { Desarrollo Sustentable } \\
c l\end{array}$ & 2016 \\
\hline 11 & $\begin{array}{c}\text { El Mercado del Diseño Arquitectónico, el } \\
\text { Desarrollo Urbano y } \\
\text { la Integración Humana }{ }^{p 2}\end{array}$ & 2016 \\
\hline 12 & Abstracciones Formales Resultantes ${ }^{p 2}$ & 2017 \\
\hline 13 & $\begin{array}{c}\text { Referentes del Segundo Resultado de Tipo } \\
\text { Histórico de la Aplicación del SIV-DAE } \\
\text { en Pachuca }{ }^{p 2}\end{array}$ & 2017 \\
\hline 14 & $\begin{array}{c}\text { Referentes del Tercer Resultado de Tipo } \\
\text { Histórico de la Aplicación del SIV-DAE } \\
\text { en Pachuca }\end{array}$ & 2017 \\
\hline 15 & $\begin{array}{c}\text { Tres Ventajas de la Aplicación del SIV- } \\
\text { DAE en Pachuca }{ }^{p 2}\end{array}$ & 2017 \\
\hline 16 & Referentes del Primer Resultado de Tipo & 2017 \\
\hline
\end{tabular}

17,18 Referentes del Cuarto Resultado de Tipo Histórico de la Aplicación del SIV-DAE en Pachuca ${ }^{p 2}$

19 La Carpeta de Trabajo del Taller de Diseño Edilicio Básico ${ }^{c l}$

20 Programación Gantt para un Curso Estratégico de Diseño Edilicio Básico ${ }^{s d}$ Competencias Medibles en el Modelado Arquitectónico Concurrente ${ }^{c l}$

Expectativas del Detonante Gráfico ${ }^{c 1}$ Evaluación de Neo Repentinas y Rendimientos para el Diseño Edilicio Básico ${ }^{c 1}$

Directrices Ambientales en el SIV-DAE de una Sucursal Bancaria de Marruecos ${ }^{p 2}$

25 Directrices Ambientales en el SIV-DAE de una Capilla Ecuménica en Cuernavaca, México ${ }^{p 2}$

26 Las Competencias Profesionalizantes

Estudiantiles y Docentes en el Diseño Edilicio Básico ${ }^{c \boldsymbol{l}}$ Arquitectónico Concurrente Detonante Gráfico $^{p l}$ Estratégico de Diseño Edilicio Básico ${ }^{p 1}$ 
Antecedentes para el Establecimiento del Mercado Correspondiente al Diseño Edilicio Básico en la Región de Pachuca ${ }^{p 1}$ Dinámicas Mercantiles en el Diseño Edilicio Básico ${ }^{s d}$

31 Programación Gantt para un Curso Estratégico de Diseño Edilicio Básico ${ }^{p 1}$ Correlatividades para el Establecimiento del Mercado Correspondiente al Diseño Edilicio Básico en la Región de Pachuca ${ }^{p 1}$ Fuente: elaboración propia.

Divulgación aunada a una serie de entrevistas accesibles en línea, planeadas para presentar y comentar amenamente, las particularidades de los cinco libros. Producidos al interior del proyecto de investigación para la Evaluación de Neo Repentinas, publicados en 2019 por la Universidad Autónoma del Estado de Hidalgo.

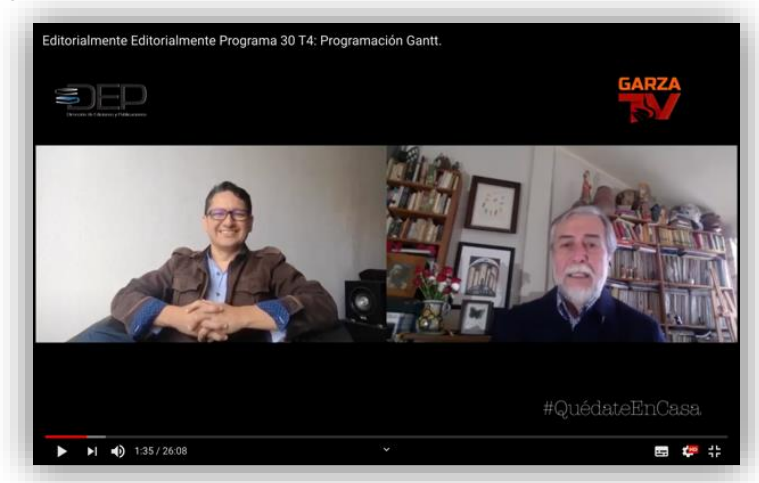

Figura 6: Imagen de una de las entrevistas; Fuente: Garza TV UAEH.

El medio digital es el programa de la misma universidad denominado "Editorialmente", coproducido por la Dirección de Ediciones y Publicaciones junto con Garza TV. El canal de Garza TV UAEH en YouTube se consigna a la promoción de los libros publicados por el personal interno dedicado a la investigación (GarzaTV-UAEH, 2020).

Tabla 3: Entrevistas/enlaces y cantidad de visualizaciones/fecha de estreno.

\begin{tabular}{|c|c|}
\hline $\begin{array}{r}\text { Título } \\
\end{array}$ & Visualizaciones \\
\hline $\begin{array}{c}\text { Modelado Arquitectónico Concurrente } \\
\text { https://www.youtube.com/watch?v=PP0ss7 } \\
\text { tZhao }\end{array}$ & $\begin{array}{c}329 \\
24 / f \mathrm{eb} / 2020\end{array}$ \\
\hline $\begin{array}{c}\text { Detonante Gráfico } \\
\text { https://www.youtube.com/watch?v=xjXpK } \\
\text { YFdN-A\&t=2s }\end{array}$ & $\begin{array}{c}\mathbf{2 5 3} \\
10 / \mathrm{mar} / 2020\end{array}$ \\
\hline $\begin{array}{c}\text { Programación Gantt para un Curso } \\
\text { Estratégico de Diseño Edilicio Básico } \\
\text { https://www.youtube.com/watch?v=21JMQ } \\
\text { I7ayOA\&t=1s }\end{array}$ & $\begin{array}{c}117 \\
14 / \mathrm{sep} / 2020\end{array}$ \\
\hline 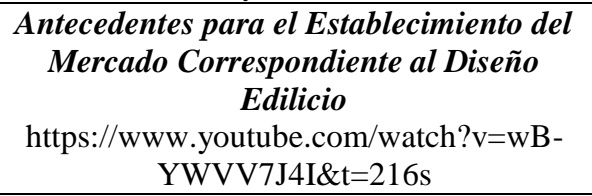 & $\begin{array}{c}118 \\
6 / \text { oct } / 2020\end{array}$ \\
\hline $\begin{array}{c}\text { Correlatividades para el Establecimiento } \\
\text { del Mercado Correspondiente al Diseño } \\
\text { Edilicio Básico en la Región de Pachuca } \\
\text { https://www.youtube.com/watch?v=YZL4 } \\
\text { WfxhlA } 4 \& \mathrm{t}=41 \mathrm{~s} \\
\end{array}$ & $\begin{array}{c}\mathbf{2 1} \\
28 / \text { oct/2020 }\end{array}$ \\
\hline
\end{tabular}

Para los libros del diseño edilicio básico se han realizado cinco entrevistas en este canal, altamente útiles para incrementar su difusión porque permite compartir los enlaces y monitorear la cantidad de sus visualizaciones, Figura 6. En la Tabla 3, se indican los títulos de las entrevistas, sus enlaces y la cantidad de visualizaciones, registradas desde sus fechas de estreno en línea a inicios del mes de noviembre del 2020.

\subsection{Los beneficios para las condiciones mercantiles del ejercicio proyectual.}

Las condiciones mercantiles del ejercicio proyectual se pueden encontrar precisadas en el libro de Antecedentes para el Establecimiento del Mercado Correspondiente al Diseño Edilicio Básico. Representadas por la definición del mercado para el diseño edilicio básico, su modelo y esquema. Ahí, el mercado se describe "como el medio exclusivo de sus profesionistas en compromiso con la concordancia de la imagen general de un conjunto urbano"; y a los protagonistas por: sus "ofertantes", "demandantes", el "producto", el "medio", la "retribución" y las "entidades vigías".

La operatividad del mercado señala "cuatro interacciones del protagonismo", previstas "para facilitar su aplicación": "económica, satisfactoria, regular y mediática". La "económica" es "propiciada por las transacciones financieras realizadas entre ofertantes y demandantes". La "satisfactoria" es representada para sus ofertantes, por la retribución un producto legal "dentro del marco arancelario", y para sus demandantes por la "obtención física o digital del producto". La "regular", es correspondiente "a los colegios de profesionistas", "gobierno estatal" y "gobiernos municipales". Consecuentemente, la "mediática" hace alusión a las "directrices regionales: territoriales y temáticas o dr:tyt" que "permiten delimitar los aspectos geográficos y tópicos particulares de una zona de estudio" (Elizalde, 2019e).

Por ende, los beneficios de las condiciones mercantiles, contemplaran esencialmente a todas aquellas oportunidades susceptibles de mejorar las "interacciones del protagonismo". Provenientes de las relaciones temáticas entre la academia de arquitectura en la UAEH, el diseño edilicio básico y el Colegio de Arquitectos de Hidalgo A.C. Bajo la premisa de señalar los elementos constitutivos de estos tres nodos, codificarlos y jerarquizarlos en términos de beneficio para las condiciones mercantiles del ejercicio proyectual agremiado en la Zona de Pachuca.

\section{Desarrollo.}

Esta indagación se ajusta a los parámetros establecidos para la investigación con enfoque cualitativo. En consecuencia es transeccional porque recopila datos en un momento dado, no es experimental ya que no maneja variables dependientes o independientes, es de alcance exploratorio debido a que trata un tema nuevo y es documental por el análisis aleatorio realizado en las fuentes consultadas. El proceso de investigación es axial y consiste en señalar a los elementos constitutivos de los tres nodos para codificarlos. Dando seguimiento a los pasos recíprocos del "diseño sistemático": (a) "recolección de los datos", (b) "codificación abierta", $(c)$ "codificación axial", $(d)$ "codificación selectiva" y $(e)$ "visualización de la teoría" (Hernández-Sampieri et al, 2014). Para finalizar en este último paso, con la hipótesis resultante, entendida como el establecimiento de la interrelación temática jerarquizada. La clasificación de los pasos con las literales de $a$, hasta $e$, fue sugerida en la presente investigación para facilitar el seguimiento de sus etapas. 
Así, la hipótesis es determinada, tanto en función del beneficio para las condiciones mercantiles del ejercicio proyectual o "interacciones del protagonismo", como por su correspondencia al diseño edilicio general y utilidad para el diseño edilicio básico; en la Zona de Pachuca.

\subsection{Etapas.}

La primera, consiste en analizar las fuentes informativas confiables de los tres nodos y tabular individualmente sus elementos constitutivos para codificarlos, implica los pasos del "diseño sistemático" clasificados con $a$ y $b$. La segunda, se concentra en observar las relaciones a partir de la aplicación del software Onodo (2020) respecto del beneficio para las condiciones mercantiles del ejercicio proyectual, comprende los pasos clasificados como $c$ y $d$. Y la tercera, se centra en presentar el mapa de relaciones temáticas entre los tres nodos, contempla el paso $e$.

\section{Elementos constitutivos de los nodos tópicos.}

Se identifican como rubros de los nodos temáticos con capacidad de beneficiar las condiciones mercantiles del ejercicio proyectual o "interacciones del protagonismo"; debido al señalamiento de su incidencia en la operatividad del mercado: económica (1), satisfactoria (2), regular (3) y mediática (4). Los nodos temáticos se precisan como: la academia de arquitectura en la UAEH, el diseño edilicio básico y el Colegio de Arquitectos de Hidalgo; codificados $(\mathrm{Cg})$ respectivamente como A, B y C. El criterio de selección consiste en identificar los rubros correspondientes al diseño edilicio en general y señalar su incidencia con una o varias de las cuatro "interacciones del protagonismo".

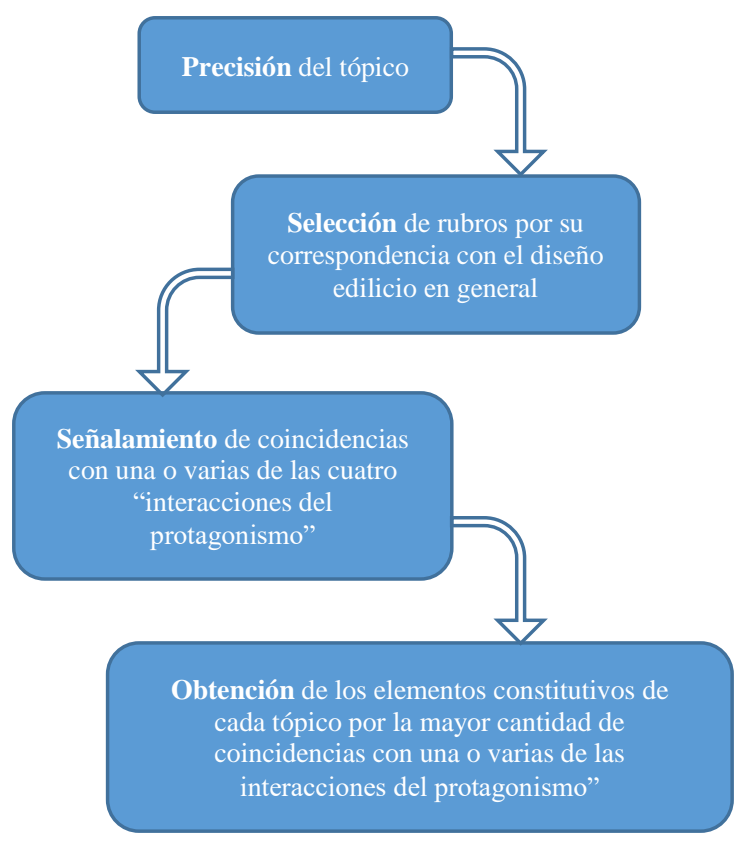

\section{Figura 7: Criterio de selección/1ra etapa; Fuente:} elaboración propia.

Por lo tanto, los elementos constitutivos de cada nodo tópico son los rubros con más coincidencias en las "interacciones del protagonismo". En la Figura 7, se observa el criterio de selección en correspondencia a la primera etapa del "diseño sistemático" de la investigación.

\subsection{En la academia de arquitectura en la UAEH.}

Los elementos constitutivos de la Academia de Arquitectura en la UAEH (A) provienen de los rubros identificados en el documento de la licenciatura (ARQ-UAEH, 2020). Fraccionado para su análisis en 9 subtemas y respetando su división de 9 semestres. En ese documento, el subtema del "campo de trabajo" se identifica como correspondiente al diseño edilicio general por referir que se puede laborar dentro de las "empresas constructoras" en "diseño arquitectónico", "en iniciativa privada como diseñador" y en instancias de gobierno atendiendo el "diseño de proyectos para beneficio social". El subtema "perfil de egreso" es correspondiente con el diseño edilicio general por precisar que poseerá aspectos cognitivos de "conceptos arquitectónicos" y "teóricos de diseño"; además de la habilidad para aplicarlos. En el mismo sentido de correspondencias con el diseño edilicio en general el subtema de "titulación" es coincidente porque hace referencia a la presentación y aprobación del "examen general de conocimientos de la licenciatura" con el "Testimonio de Desempeño Satisfactorio".

Tabla 4: Elementos constitutivos de la academia de arquitectura en la UAEH.

\begin{tabular}{|c|c|c|c|c|c|}
\hline \multirow{2}{*}{$C g$} & \multirow{2}{*}{ Rubros } & \multicolumn{4}{|c|}{ Incidencias } \\
\hline & & 1 & 2 & 3 & 4 \\
\hline $1 \mathrm{~A}$ & Campo de trabajo & $\mathrm{x}$ & & & \\
\hline $2 \mathrm{~A}$ & Perfil de egreso & & & & $\mathrm{x}$ \\
\hline $3 \mathrm{~A}$ & Titulación & & & & $\mathrm{x}$ \\
\hline $4 \mathrm{~A}$ & 1erSem, Metodología del diseño & $\mathrm{x}$ & $\mathrm{x}$ & & \\
\hline $5 \mathrm{~A}$ & $\begin{array}{l}\text { 2doSem, Fundamentos teóricos } \\
\text { del diseño }\end{array}$ & $\mathrm{x}$ & $\mathrm{x}$ & & \\
\hline 6A & $\begin{array}{l}\text { 3erSem, Diseño arquitectónico } \\
\text { elemental }\end{array}$ & $\mathrm{x}$ & $\mathrm{x}$ & & $\mathrm{x}$ \\
\hline 7A & $\begin{array}{l}\text { 4toSem, Diseño arquitectónico } \\
\text { de actividades interpersonales }\end{array}$ & $\mathrm{x}$ & $\mathrm{x}$ & & $\mathrm{x}$ \\
\hline $\mathbf{8 A}$ & $\begin{array}{l}\text { 5toSem, Diseño e intervención } \\
\text { arquitectónica }\end{array}$ & $\mathrm{x}$ & $\mathrm{x}$ & & $\mathrm{x}$ \\
\hline $\mathbf{9 A}$ & $\begin{array}{l}\text { 6toSem, Diseño arquitectónico } \\
\text { de actividades }\end{array}$ & $\mathrm{x}$ & $\mathrm{x}$ & & $\mathrm{x}$ \\
\hline $10 \mathrm{~A}$ & $\begin{array}{l}7 \text { moSem, Diseño arquitectónico } \\
\text { de impacto regional }\end{array}$ & $\mathrm{x}$ & $\mathrm{x}$ & & \\
\hline $11 \mathrm{~A}$ & $\begin{array}{l}7 \text { moSem, Diseño aplicado al } \\
\text { paisaje y medio ecológico }\end{array}$ & $\mathrm{x}$ & $\mathrm{x}$ & & \\
\hline $12 \mathrm{~A}$ & $\begin{array}{l}\text { 8voSem, Diseño arquitectónico e } \\
\text { intervención urbana }\end{array}$ & $\mathrm{x}$ & $\mathrm{x}$ & & \\
\hline $13 \mathrm{~A}$ & $\begin{array}{l}\text { 8voSem, Diseño de espacios para } \\
\text { necesidades sociales }\end{array}$ & $\mathrm{x}$ & $\mathrm{x}$ & & \\
\hline $14 \mathrm{~A}$ & 9noSem, Servicio social & & & $\mathrm{x}$ & \\
\hline $15 \mathrm{~A}$ & $\begin{array}{l}\text { 9noSem, Mercadotecnia en la } \\
\text { arquitectura }\end{array}$ & $\mathrm{x}$ & & & \\
\hline $16 \mathrm{~A}$ & $\begin{array}{l}\text { 9noSem, Diseño de mobiliario } \\
\text { multifuncional }\end{array}$ & $\mathrm{x}$ & & & \\
\hline $17 \mathrm{~A}$ & $\begin{array}{l}\text { 10moSem, Prácticas } \\
\text { profesionales }\end{array}$ & & & $\mathrm{x}$ & \\
\hline $18 \mathrm{~A}$ & $\begin{array}{l}\text { 10moSem, arquitectura para } \\
\text { necesidades emergentes y } \\
\text { efímeras }\end{array}$ & & & & $\mathrm{x}$ \\
\hline 19A & $\begin{array}{l}\text { 10moSem, diseño de espacio de } \\
\text { interiores }\end{array}$ & & $\mathrm{x}$ & & \\
\hline
\end{tabular}

De igual modo, el "primer semestre" es coincidente con el diseño edilicio en general debido a la asignatura "metodología de la investigación"; el "segundo semestre" por la asignatura "fundamentos teóricos de diseño"; el "tercer semestre" por la 
asignatura "diseño arquitectónico elemental"; el "cuarto semestre" por la asignatura "diseño arquitectónico de actividades interpersonales"; el "quinto semestre" por la asignatura "diseño arquitectónico de actividades"; el "séptimo semestre" por las asignaturas "diseño arquitectónico de impacto regional” y "diseño aplicado al paisaje y medio ecológico".

El "octavo semestre por la asignatura "diseño arquitectónico e intervención urbana" y la "optativa I" de "diseño de espacios para necesidades sociales"; el "noveno semestre" por el subtema "servicio social" y las asignaturas de la "optativa II" indicadas como "mercadotecnia en la arquitectura" y "diseño de mobiliario multifuncional". Así el "décimo semestre" es coincidente por el subtema de "prácticas profesionales" y por las asignaturas "arquitectura para necesidades emergentes y efímeras" de la "optativa III", además de la asignatura de "diseño de espacio de interiores" de la "optativa IV".

El subtema del "servicio social" coincide al mencionar la "participación activa" del estudiantado "en la solución de problemas específicos que le permiten el desarrollo de una conciencia social". La "práctica profesional" también es coincidente la enunciar la inclusión del estudiantado "en el sector productivo, social y privado" para conocer "la realidad socioeconómica y profesional".

Una vez realizado el señalamiento de las coincidencias, los elementos constitutivos de la academia de arquitectura en la UAEH son: La asignatura de "diseño arquitectónico elemental" del tercer semestre, codificada como 6A-1,2,4. La asignatura de "diseño arquitectónico de actividades interpersonales" del cuarto semestre, codificada como 7A-1,2,4. La asignatura de "diseño e intervención arquitectónica" del quinto semestre, codificada como $\mathbf{8 A - 1 , 2 , 4}$. Y la asignatura de "diseño arquitectónico de actividades" del sexto semestre, codificada como 9A-1,2,4, Tabla 4.

\subsection{En el diseño edilicio básico.}

Los elementos constitutivos del diseño edilicio básico (B) provienen de los rubros, representados por las 21 tipologías, identificados en el documento titulado "La Importancia de la Evaluación Nacional de las Competencias Profesionalizantes del Diseño Edilicio Básico" (Elizalde, 2017). Alojado para el alcance del estudiantado y procurar su consulta o lectura, en el repositorio de la Universidad Autónoma del Estado de Hidalgo, abreviado como RIA-UAEH.

Las tipologías se clasifican en el arancel del Colegio de Arquitectos por su código $\left.{ }^{c c 1}\right)$ y "factor de costo" $\left({ }^{c c 2}\right)$. El valor de este factor se identifica como el indicador de la correspondencia al diseño edilicio general. En esa perspectiva, se puede decir que a mayor "factor de costo" $\left({ }^{c c 2}\right)$, mayor correspondencia con el diseño edilicio general, y viceversa. Al observar los factores de las 21 tipologías del documento de origen, destacan los valores reiterados de 1.04 repetido en dos ocasiones, el valor de 1.45 en tres ocasiones y el valor de 2.07 en tres ocasiones.

Por consiguiente, los "dispensarios médicos" y “almacenes" se vuelven correspondientes al diseño edilicio general debido a que propician la doble repetición del "factor de costo" (cc2) con valor de 1.04. Las "cafeterías", "neverías" y "almacenes" corresponden al diseño edilicio general porque poseen el "factor de costo" $\left({ }^{c c 2}\right)$ con valor de 1.45 repetido en 3 ocasiones. Asimismo, las "boutiques", "joyerías" y tiendas especializadas" son correspondientes con el diseño edilicio general por la triple repetición del "factor de costo" $\left({ }^{c c 2}\right)$ con valor de 2.07 .

Realizado el señalamiento de las coincidencias, los elementos constitutivos del diseño edilicio básico son: Las "joyerías" con "factor de costo" $\left({ }^{c c 2}\right)$ igual a 1.45 , codificadas como $\mathbf{2 B - 1 , 2 , 4}$. Las "neverías" con "factor de costo" ( $\left.{ }^{c c 2}\right)$ igual a 1.45 , codificadas como 3B-1,2,4. Incluidas las "tiendas especializadas" con "factor de costo" ${ }^{c c 2}$ ) igual a 1.45 , codificadas como 7B-1,2,4. Para asignar la primera incidencia a las tres tipologías resultantes se estimó que son más comunes en el tejido urbano sobre el resto de las 21 tipologías, Tabla 5.

Tabla 5: Elementos constitutivos del diseño edilicio básico.

\begin{tabular}{|c|c|c|c|c|c|}
\hline \multirow{2}{*}{$C g$} & \multirow{2}{*}{ Rubros } & \multicolumn{4}{|c|}{ Incidencias } \\
\hline & & 1 & 2 & 3 & 4 \\
\hline 1B & $\begin{array}{l}\text { Agencias de autos (B-1 }{ }^{c c 1}, \\
1.45^{c c 2} \text { ) }\end{array}$ & & $\mathrm{x}$ & & $\mathrm{x}$ \\
\hline $2 B$ & Cafeterías (Q-2 ${ }^{c c 1}, 1.45^{c c 2}$ ) & $\mathrm{x}$ & $\mathrm{x}$ & & $\mathrm{x}$ \\
\hline 3B & Neverías $\left(\mathrm{Q}-2^{c c 1}, 1.45^{c c 2}\right)$ & $\mathrm{x}$ & $\mathrm{x}$ & & $\mathrm{X}$ \\
\hline 4B & Boutiques $\left(\mathrm{B}-14^{c c 1}, 2.07^{c c 2}\right)$ & & $\mathrm{x}$ & & $\mathrm{x}$ \\
\hline $5 B$ & $\begin{array}{l}\text { Dispensarios médicos }\left(\mathrm{O}-5^{c c 1},\right. \\
\left.1.04^{c c 2}\right)\end{array}$ & & $\mathrm{x}$ & & $\mathrm{x}$ \\
\hline $6 \mathrm{~B}$ & Joyerías $\left(\mathrm{B}-8^{c c 1}, 2.07^{c c 2}\right)$ & & $\mathrm{X}$ & & $\mathrm{x}$ \\
\hline $7 \mathbf{B}$ & $\begin{array}{l}\text { Tiendas especializadas (B-14 }{ }^{c c 1} \text {, } \\
2.07^{c c 2} \text { ) }\end{array}$ & $\mathrm{x}$ & $\mathrm{x}$ & & $\mathrm{x}$ \\
\hline $8 \mathrm{~B}$ & Almacenes $\left(\mathrm{B}-2^{c c 1}, 1.04^{c c 2}\right)$ & & $\mathrm{X}$ & & $\mathrm{X}$ \\
\hline
\end{tabular}

\subsection{En el Colegio de Arquitectos de Hidalgo.}

Los elementos constitutivos del Colegio de Arquitectos de Hidalgo (C), provienen de los rubros, representados por las parcialidades de los productos de las primeras tres etapas de los servicios del arancel del Colegio de Arquitectos de Hidalgo A.C. (ECA-CAH, 2012).

Tabla 6: Elementos constitutivos del Colegio de Arquitectos de Hidalgo.

\begin{tabular}{|c|c|c|c|c|c|}
\hline \multirow{2}{*}{$C g$} & \multirow{2}{*}{ Rubros } & \multicolumn{4}{|c|}{ Incidencias } \\
\hline & & 1 & 2 & 3 & 4 \\
\hline $1 \mathrm{C}$ & $\begin{array}{l}\text { I.1; Planos Arquitectónicos } \\
(11 \% / 7=1.57 \%)\end{array}$ & $\mathrm{x}$ & $\mathrm{x}$ & & $\mathrm{x}$ \\
\hline $2 \mathrm{C}$ & $\begin{array}{l}\text { I.1; Memoria expositiva del } \\
\text { concepto arquitectónico } \\
(11 \% / 7=1.57 \%)\end{array}$ & & $\mathrm{x}$ & & \\
\hline $3 \mathrm{C}$ & $\begin{array}{l}\text { I. } 1 ; \text { Esquema funcional } \\
(11 \% / 7=1.57 \%)\end{array}$ & & $\mathrm{x}$ & & \\
\hline $4 C$ & $\begin{array}{l}\text { I.1; Imagen conceptual o } \\
\text { perspectivas volumétricas } \\
(11 \% / 7=1.57 \%)\end{array}$ & $\mathrm{x}$ & $\mathrm{x}$ & & $\mathrm{x}$ \\
\hline $5 \mathrm{C}$ & $\begin{array}{l}\text { I. } 2 \text {; Memoria descriptiva del } \\
\text { proyecto }(20 \% / 8=2.5 \%)\end{array}$ & & $\mathrm{x}$ & & \\
\hline $6 \mathrm{C}$ & $\begin{array}{l}\text { I. } 2 \text {; Plantas cortes y fachadas a } \\
\text { escala }(20 \% / 8=2.5 \%)\end{array}$ & $\mathrm{x}$ & $\mathrm{x}$ & & $\mathrm{x}$ \\
\hline $7 \mathrm{C}$ & $\begin{array}{l}\text { I. } 2 \text {; Apuntes en perspectiva } \\
(20 \% / 8=2.5 \%)\end{array}$ & $\mathrm{x}$ & $\mathrm{x}$ & & $\mathrm{x}$ \\
\hline $8 C$ & $\begin{array}{l}\text { I.3; Planos arquitectónicos } \\
\text { detallados }(35 \% / 9=3.88 \%)\end{array}$ & $\mathrm{x}$ & $\mathrm{x}$ & & $\mathrm{x}$ \\
\hline $9 \mathrm{C}$ & $\begin{array}{l}\text { I.3; Detalles constructivos } \\
(35 \% / 9=3.88 \%)\end{array}$ & & $\mathrm{x}$ & & \\
\hline $10 \mathrm{C}$ & $\begin{array}{l}\text { I.3; Planos detallados de herrería } \\
\text { y/o cancelería y/o carpintería } \\
(35 \% / 9=3.88 \%)\end{array}$ & & $\mathrm{x}$ & & \\
\hline $11 \mathrm{C}$ & $\begin{array}{l}\text { I.3; Planos de albañilería } \\
(35 \% / 9=3.88 \%)\end{array}$ & & $\mathrm{x}$ & & \\
\hline $12 \mathrm{C}$ & $\begin{array}{l}\text { I.3; Perspectivas detalladas } \\
(35 \% / 9=3.88 \%)\end{array}$ & & $\mathrm{x}$ & & \\
\hline
\end{tabular}


El apartado de "Productos y Participación por Servicios Parciales", del documento de partida, se divide en 7 etapas, las primeras tres conciernen directamente a las actividades profesionales del proyecto arquitectónico. Indicadas como "I.1.Diseño Conceptual", comprende 7 productos y le es propio el 11\% de participación. La siguiente es la etapa "I.2.- Anteproyecto", comprende 8 productos y le es propio el $20 \%$ de participación. La última es la etapa "I.3.- Diseño Ejecutivo", comprende 9 productos y le es propio el 35\% de participación.

Sin omisión alguna los planos y perspectivas se consideran como los mejores descriptores del proyecto. Así, los "planos arquitectónicos" y la "imagen conceptual" de la etapa I.1. con valor de $1.57 \%$ respectivamente son correspondientes con el diseño edilicio general. Las "plantas cortes y fachadas a escala" aunados a los "apuntes en perspectiva" con valores respectivos de $2.5 \%$ también son correspondientes con el diseño edilicio general. Por último, los "planos arquitectónicos detallados" con valor de $3.8 \%$ de igual modo corresponden al diseño edilicio general.

En términos específicos, los "planos arquitectónicos" se clasifican como 1C-1,2,4; la "imagen conceptual" como 4C-1,2,4; las "plantas cortes y fachadas a escala" como 6C-1,2,4; los "apuntes en perspectiva" como 7C-1,2,4; y los "planos arquitectónicos detallados" como $\mathbf{8 C - 1 , 2 , 4}$. Las incidencias 1,2 y 4 se vuelven constantes porque se aprecia su repercusión continua en el medio profesional. Menor a la solicitud del resto de los productos, Tabla 6.

\section{Resultados.}

Se presentan en cuatro aspectos: la red de los nodos con sus elementos constitutivos, la relación temática definitiva entre los tres nodos, el mapa de relaciones temáticas y la expresión de la hipótesis resultante.

\subsection{Red de los nodos con sus elementos constitutivos.}

En la Figura 8, se presentan los tres nodos y sus elementos constitutivos. Fue elaborada con uso del software Onodo y permite distinguir las relaciones primarias entre los nodos y secundarias de estos con elementos constitutivos respectivos.

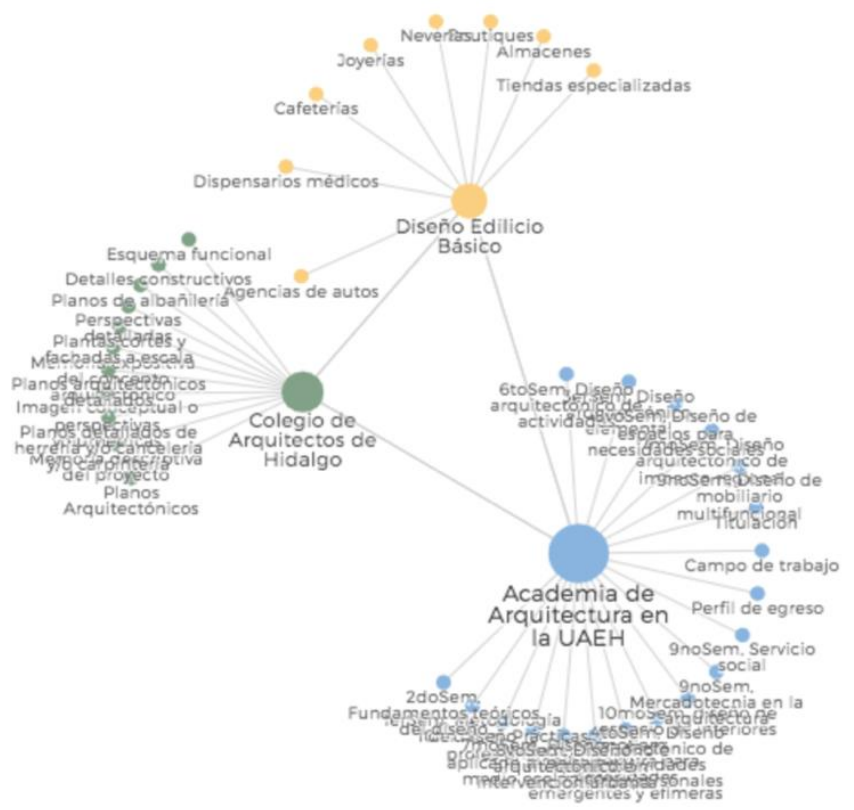

Figura 8: Nodos y elementos constitutivos; Fuente: elaboración propia.

\subsection{Tabla de relaciones temáticas.}

La relación temática definitiva ( $\boldsymbol{R} t \boldsymbol{t})$ se establece en consideración a la utilidad de los elementos constitutivos de los nodos de la academia de arquitectura en la UAEH y del Colegio de Arquitectos de Hidalgo para proyectar los elementos constitutivos del nodo asignado al diseño edilicio básico, Tabla 7.

Finalmente la Rtd queda determinada por el "diseño arquitectónico de actividades interpersonales codificado como 7A-1,2,4 del nodo para la academia de arquitectura en la UEAH, así como por las "plantas cortes y fachadas a escala" codificadas como 6C-1,2,4 para el nodo del Colegio de Arquitectos de Hidalgo. Ambas relaciones en correspondencia al nodo del diseño edilicio básico, dispuestas a partir de las "joyerías" codificadas como 2B-1,2,4; las "neverías" codificadas como $\mathbf{3 B - 1 , 2 , 4}$ y las "tiendas especializadas" codificadas como 7B-1,2,4.

Tabla 7: Relaciones temáticas definitivas

\begin{tabular}{|c|c|c|}
\hline $\mathrm{Cg}$ & Nodos/Elementos Constitutivos & Rtd \\
\hline \multicolumn{3}{|c|}{ Academia de Arquitectura en la UAEH } \\
\hline $6 \mathrm{~A}-1,2,4$ & Diseño arquitectónico elemental & \\
\hline $7 \mathrm{~A}-1,2,4$ & $\begin{array}{l}\text { Diseño arquitectónico de actividades } \\
\text { interpersonales }\end{array}$ & $\mathrm{x}$ \\
\hline $8 \mathrm{~A}-1,2,4$ & Diseño e intervención arquitectónica & \\
\hline $9 \mathrm{~A}-1,2,4$ & Diseño arquitectónico de actividades & \\
\hline \multicolumn{3}{|c|}{ diseño edilicio básico } \\
\hline 2B-1,2,4 & Joyerías & $\mathrm{x}$ \\
\hline $3 B-1,2,4$ & Neverías & $\mathrm{x}$ \\
\hline 7B-1,2,4 & Tiendas especializadas & $\mathrm{x}$ \\
\hline \multicolumn{3}{|c|}{ Colegio de Arquitectos de Hidalgo } \\
\hline $1 \mathrm{C}-1,2,4$ & Planos arquitectónicos & \\
\hline $4 C-1,2,4$ & Imagen conceptual & \\
\hline $6 C-1,2,4$ & Plantas cortes y fachadas a escala & $\mathrm{x}$ \\
\hline $7 C-1,2,4$ & Apuntes en perspectiva" & \\
\hline $8 C-1,2,4$ & Planos arquitectónicos detallados & \\
\hline
\end{tabular}

\subsection{Mapa de relaciones temáticas.}

La expresión gráfica de las relaciones temáticas definitivas de la academia en la UAEH y del Colegio de Arquitectos, respecto de sus elementos constitutivos, se muestra en la Figura 9.

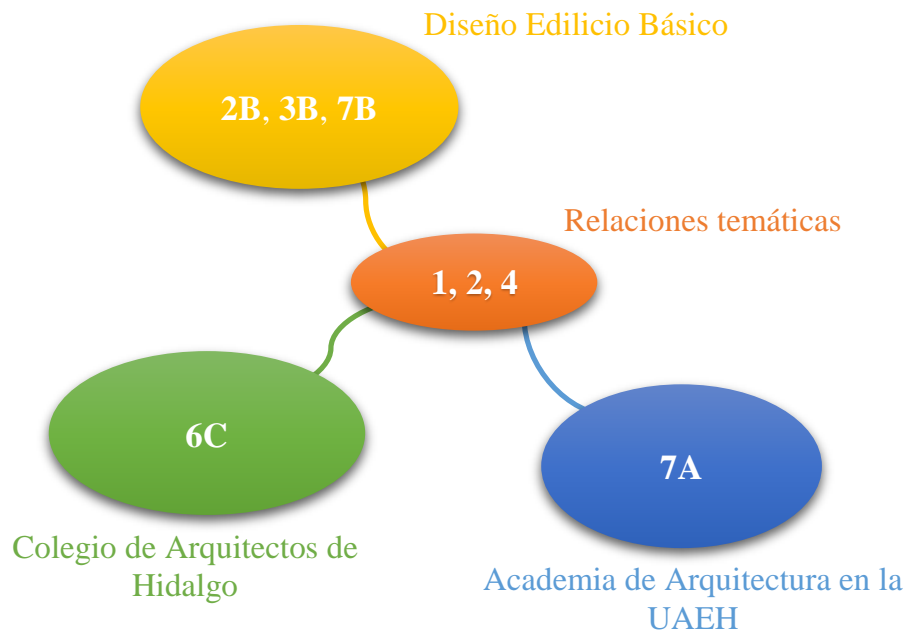

Figura 9: Mapa de relaciones temáticas; Fuente: elaboración propia. 
Útil para proyectar a los elementos constitutivos del nodo relativo al diseño edilicio básico. Tercia de elementos ligados por la prevalencia de las "interacciones del protagonismo" económica (1), satisfactoria (2) y mediática (4).

\subsection{Expresión de la hipótesis resultante.}

Las aproximaciones temáticas se encuentran determinadas por 3 de las 4 "interacciones del protagonismo" económica (1), satisfactoria (2) y mediática (4). Observadas como constantes correlativas de los elementos constitutivos en el "diseño arquitectónico de actividades interpersonales codificado para el nodo de la academia de arquitectura en la UEAH como 7A-1,2,4. Igualmente constantes en las "plantas cortes y fachadas a escala" codificadas para el nodo del Colegio de Arquitectos de Hidalgo como 6C-1,2,4. Además de las "joyerías" codificadas como 2B$\mathbf{1 , 2 , 4}$; las "neverías" codificadas como $\mathbf{3 B}-\mathbf{1 , 2 , 4}$ y las "tiendas especializadas" codificadas como $\mathbf{7 B - 1 , 2 , 4}$ para el nodo del diseño edilicio básico. Atributos suficientes para considerar a estos 5 elementos constitutivos como rubros con capacidad de beneficiar las condiciones mercantiles del ejercicio proyectual o "interacciones del protagonismo".

\section{Discusión.}

Si bien esta hipótesis resultante es un punto de partida para futuras investigaciones, es importante constatar que, a pesar de la contundencia impuesta en su afirmación, para estimar la capacidad de beneficio en los 5 rubros; proveniente del cabal cumplimiento concerniente al procedimiento contenido en la investigación con enfoque cualitativo. Esta afirmación no deja de ser una tentativa de solución utilizable para estrechar la distancia de desconocimiento y desentendimiento de la ejecución proyectual existente entre la formación académica y desempeño profesional. Por ello debe tomarse con las reservas que su ubicación local le confiere, pero al mismo tiempo, sin dejar de considerarle como argumento aplicable y replicable en otros tejidos urbanos.

Consecuentemente, en materia de proyectos con incidencia en los parámetros nacionales relativos a la estructura universal del Arancel de la FCARM y los establecidos para el gremio por entidades con repercusión internacional como el Consejo Nacional de Registro de la Certificación Profesional de Arquitectos (CONARC, 2021).

\section{Conclusiones.}

La estructura gráfica del mapa permite el uso y aplicación de éstas relaciones temáticas en la toma de conciencia, decisiones y gestión de directivas; concernientes a la generalidad local de las entidades educativas, de investigación y del gremio. Asimismo al respecto del presente y del futuro de las oportunidades de ingreso financiero; propias del empleo y autoempleo en la Zona de Pachuca. Porqué proviene de un trabajo de investigación con enfoque cualitativo. Proceso que le confiere el carácter de confiable y verificable.

\section{Agradecimientos}

En la Universidad Autónoma del Estado de Hidalgo, por el apoyo y las facilidades otorgadas para realizar el presente trabajo de investigación, a la jefatura del Área Académica de Ingeniería y Arquitectura; a la dirección del Instituto de Ciencias Básicas e Ingeniería; y a la coordinación del programa educativo de la licenciatura en Arquitectura. Así como al Consejo Editorial Universitario, a Garza TV de la UAEH y al programa Editorialmente. Extracadémicamente al Despacho de Arquitectura CED y al Colegio de Arquitectos de Hidalgo A.C.; por el apoyo recibido.

\section{Referencias}

AAIyA, (2020). Área Académica de Ingeniería y Arquitectura, Instituto de ciencias Básica e Ingeniería. Sitio consultado el 6 noviembre del 2020 en https://www.uaeh.edu.mx/campus/icbi/investigacion/ingenieria/index.htm

ARQ-UAEH. (2020). Licenciatura en Arquitectura. Universidad Autónoma del Estado de Hidalgo. Recuperado el 8 noviembre del 2020 de https://www.uaeh.edu.mx/campus/icbi/oferta/licenciaturas/lic_arquitectur a.html

CAH. (2020). Colegio de Arquitectos del Estado de Hidalgo A.C. Sitio consultado el 7 de noviembre del 2020 en http://www.cah.com.mx/

CONARC. (2021). Consejo Nacional de Registro de la Certificación Profesional de Arquitectos. Sitio consultado el 2 de enero del 2021 en https://www.fcarm.org.mx/convocatorias-conarc/

ECA-CAH. (2012). Estatuto, Código de Ética, Arancel. Colegio de Arquitectos de Hidalgo A.C.

Elizalde, Domínguez, Continente (2020). Diseño Edilicio Básico, definición y productos al 2019. Pädi. Vol. 7 No. 14 (2020) 66-73. DOI: https://doi.org/10.29057/icbi.v7i14.5045

Elizalde, Domínguez, Continente. (2017). La Importancia de la Evaluación Nacional de las Competencias Profesionalizantes del Diseño Edilicio Básico. Repositorio de la Universidad Autónoma del Estado de Hidalgo. RIA-UAEH. Recuperado el 9 noviembre del 2020 de https://repository.uaeh.edu.mx/bitstream/handle/123456789/19222

Elizalde, Domínguez, Continente. (2019a). Correlatividades para el Establecimiento del Mercado Correspondiente al Diseño Edilicio Básico en la Región de Pachuca. Universidad Autónoma del Estado de Hidalgo. Biblioteca Digital. Recuperado el 6 noviembre del 2020 de http://dgsa.uaeh.edu.mx:8080/bibliotecadigital/handle/231104/2093

Elizalde, Domínguez, Continente. (2019b). Modelado Arquitectónico Concurrente. Universidad Autónoma del Estado de Hidalgo. Biblioteca Digital.

http://dgsa.uaeh.edu.mx:8080/bibliotecadigital/handle/231104/2097

Elizalde, Domínguez, Continente. (2019c). Donante Gráfico. Universidad Autónoma del Estado de Hidalgo. Biblioteca Digital. http://dgsa.uaeh.edu.mx:8080/bibliotecadigital/handle/231104/2096

Elizalde, Domínguez, Continente. (2019d). Programación Gantt para un Curso Estratégico de Diseño Edilicio Básico. Universidad Autónoma del Estado de Hidalgo. Biblioteca Digital http://dgsa.uaeh.edu.mx:8080/bibliotecadigital/handle/231104/2095

Elizalde, Domínguez, Continente. (2019e). Antecedentes para el Establecimiento del Mercado Correspondiente al Diseño Edilicio Básico en la Región de Pachuca. Universidad Autónoma del Estado de Hidalgo. Biblioteca Digital. Recuperado el 8 noviembre del 2020 de http://dgsa.uaeh.edu.mx:8080/bibliotecadigital/handle/231104/2094

FCARM. (2020). Federación de Colegios de Arquitectos de la República Mexicana A.C. Sitio consultado el 7 noviembre del 2020 en http://www.fcarm.org.mx/aranceles/

GarzaTV-UAEH. (2020). Garza TV UAEH. Canal de YouTube. Sitio $\begin{array}{llllll}\text { consultado el } & 8 & \text { noviembre del } & 2020 & \text { en }\end{array}$ https://www.youtube.com/c/GarzaTVUAEH/videos

Hernández-Sampieri R., Fernández-Collado R., Baptista-Lucio P, (2014), Metodología de la Investigación, México, Mc Graw Hill. 6ta Edición. El oso panda.com. Recuperado el 8 noviembre del 2020 de https://www.uca.ac.cr/wp-content/uploads/2017/10/Investigacion.pdf

INEGI. (1991). Datos Básicos de la Geografía de México. Instituto Nacional de Estadística y Geografía. México. Cuadro 1.2.h. pp. 20. Recuperado el 6 noviembre del $2020 \quad$ de http://internet.contenidos.inegi.org.mx/contenidos/productos/prod_serv/c ontenidos/espanol/bvinegi/productos/historicos/2104/702825221218/702 825221218_1.pdf

INEGI. (2009a). Prontuario de información geográfica municipal de los Estados Unidos Mexicanos. Pachuca de Soto, Hidalgo. Instituto Nacional de Estadística y Geografía. México. Clave geoestadística 13048. Recuperado el 6 noviembre del 2020 de http://www3.inegi.org.mx/contenidos/app/mexicocifras/datos_geografico s/13/13048.pdf 
INEGI. (2009b). Prontuario de información geográfica municipal de los Estados Unidos Mexicanos. Mineral de la Reforma, Hidalgo. Instituto Nacional de Estadística y Geografía. México. Clave geoestadística 13051. Recuperado el 6 noviembre del 2020 de https://www.inegi.org.mx/contenidos/app/mexicocifras/datos_geograficos /13/13051.pdf

INEGI. (2009c). Prontuario de información geográfica municipal de los Estados Unidos Mexicanos. Mineral del Monte, Hidalgo. Instituto Nacional de Estadística y Geografía. México. Clave geoestadística 13039. Recuperado el 6 noviembre del 2020 de http://www3.inegi.org.mx/contenidos/app/mexicocifras/datos_geografico s/13/13039.pdf

INEGI. (2009d). Prontuario de información geográfica municipal de los Estados Unidos Mexicanos. Mineral del Chico, Hidalgo. Instituto Nacional de Estadística y Geografía. México. Clave geoestadística 13038. Recuperado el 6 noviembre del 2020 de http://www3.inegi.org.mx/contenidos/app/mexicocifras/datos_geografico s/13/13038.pdf

INEGI. (2009e). Prontuario de información geográfica municipal de los Estados Unidos Mexicanos. Zempoala. Hidalgo. Instituto Nacional de Estadística y Geografía. México. Clave geoestadística 13082. Recuperado el 6 noviembre del 2020 de https://www.inegi.org.mx/contenidos/app/mexicocifras/datos_geograficos /13/13083.pdf

INEGI. (2009f). Prontuario de información geográfica municipal de los Estados Unidos Mexicanos. Zapotlán de Juárez. Hidalgo. Instituto Nacional de Estadística y Geografía. México. Clave geoestadística 13082. Recuperado el 6 noviembre del 2020 de http://www3.inegi.org.mx/contenidos/app/mexicocifras/datos_geografico s/13/13082.pdf

INEGI. (2009g). Prontuario de información geográfica municipal de los Estados Unidos Mexicanos. San Agustín Tlaxica. Hidalgo. Instituto Nacional de Estadística y Geografía. México. Clave geoestadística 13052. Recuperado el 6 noviembre del 2020 de http://www3.inegi.org.mx/contenidos/app/mexicocifras/datos_geografico s/13/13052.pdf

INEGI. (2016). Anuario estadístico y geográfico de Hidalgo 2017. Instituto Nacional de Estadística y Geografía. México. Cuadro 18.3. Recuperado el 6 noviembre del 2020 de http://internet.contenidos.inegi.org.mx/contenidos/Productos/prod_serv/c ontenidos/espanol/bvinegi/productos/nueva_estruc/anuarios_2017/70282 5095093.pdf

INEGI. (2020). Información para niños, resumen. Instituto Nacional de Estadística y Geografía. México. Sitio consultado el 6 noviembre del 2020 de http://cuentame.inegi.org.mx/monografias/informacion/hgo/

LAHDUOTEH. (2018). Ley de Asentamientos Humanos, Desarrollo Urbano y Ordenamiento Territorial del Estado de Hidalgo. Recuperada el 2 de enero del 2021 en: http://www.congresohidalgo.gob.mx/biblioteca_legislativa/Leyes/16Ley\%20de\%20Asentamie ntos $\% 20$ Humanos, $\% 20$ Desarrollo\%20Urbano\%20y\%20Ordenamiento\% 20Territorial.pdf

LEPEH. (2001). Ley del Ejercicio Profesional para el Estado de Hidalgo. Recuperada el 2 de enero del 2021 en: https://docs.mexico.justia.com/estatales/hidalgo/ley-del-ejercicioprofesional-para-el-estado-de-hidalgo.pdf

Marrero Sánchez, Odalys. Mohamed Amar, Rachida. Xifra Triadú, Jordi. (2018). "Habilidades Blandas: Necesarias para la Formación Integral del Estudiante Universario". Ecociencia. Recuperado el 2 de enero del 2021 en: file:///Users/contyiii/Downloads/144 Texto\%20de1\%20art\%C3\%ADculo-221-1-10-20190815.pdf

Onodo. (2020). Software on line especializado en la elaboración de redes. Sitio consultado el 10 noviembre del 2020 en https://onodo.org/

ONU-HABITAT. (2016). Pachuca de Soto, Hidalgo, México. Índice de Básico de las Ciudades Prósperas. México. Organización de las Naciones Unidas. pp. 17-.19. Recuperado el 6 noviembre del 2020 de https://cpi.unhabitat.org/sites/default/files/resources/HID_Pachuca_de_So to.pdf

SEDATU-CONAPO-INEGI. (2018). Delimitación de las zonas metropolitanas de México 2015. Secretaría de Desarrollo Agrario, Territorial y Urbano, Consejo Nacional de Población, Instituto Nacional de Estadística y Geografía. México. pp. 1130-132. Recuperado el 7 noviembre del 2020 de https://www.gob.mx/cms/uploads/attachment/file/305634/Delimitacion_Z onas_Metropolitanas_2015.pdf

Tito Maya, María D. Serrano Orellena, Bill. (2016). "Desarrollo de sotd skills una alternativa a la escasez de talento humano". Innova. Vol. 1, No. 12, pp. 59-76. DOI: https://doi.org/10.33890/innova.v1.12.2016.81 